\title{
Preserving Foot Length: Pan-Digital Amputation an Alternative Amputation to Transmetatarsal Amputation: A Case Study
}

\author{
${ }^{1}$ Resident Physician, Medstar Georgetown University Hospital, USA \\ ${ }^{2}$ Attending Physician, Medstar Franklin Square Medical Center, USA \\ ${ }^{3}$ Attending Physician, Orthopedic Associates of Lake County, USA
}

Jered $M$ Stowers, $D P M^{1^{*}}$, Paul Carroll, $D P M^{2}$ and Jonathon J Sharpe, DPM ${ }^{3}$

*Corresponding author: Jered M Stowers, DPM, Resident Physician, Medstar Georgetown University Hospital, Washington, DC, USA

\begin{abstract}
Maintaining foot length during limb salvage procedures is of paramount importance to maintaining foot function. Currently, the most clinically accepted amputation for an unsalvageable forefoot is the transmetatarsal amputation. While this procedure has predictable results, there are pitfalls to the procedure. Under certain circumstances, a pan-digital amputation may be a more functional amputation. There is limited literature on outcomes of this procedure. The case presented highlights a patient who underwent a successful pan-digital amputation.
\end{abstract}

\section{Keywords}

Limb salvage, Digital amputation, Infection, Forefoot amputation, Osteomyelitis

\section{Introduction}

In the Diabetic population, $12-25 \%$ are at risk of developing an ulceration $[1,2]$. Approximately $11 \%$ of individuals who have a diabetic ulceration go on to have an amputation [2]. Diabetes related amputations account for $40-65 \%$ of all non-traumatic lower extremity amputations $[1,3]$.

Lateral lesser digit amputation has a relatively low impact on ambulation [4]. If multiple lesser digits, central digits, or hallux amputations are performed, however, this can lead to digital deviation or rigid contracture of the toes [4]. This can result in new sites of ulcerations and infection. For prevention of these deformities, many surgeons prefer to perform a transmetatarsal amputation (TMA). The TMA has been a reliable amputation to maintain limb length; however, due to osteotomies through the metatarsals it has the potential to increase forefoot pressure if the metatarsal parabola is not re-established [2]. Mueller, et al. reported increased peak plantar of the forefoot and decreased peak pressures on the heel with patients who underwent a TMA [5]. In the same study, he observed a $27 \%$ skin breakdown in 102 patients following a TMA [5].

It has been established that proximal amputations require considerably more energy during ambulation [46]. In regards to forefoot amputations, this deficit is the result of a shorter lever arm and the decrease in plantar flexory power of the ankle during gait [7]. Amputation proximal to the metatarsophalangeal joint (MPTJ) has been shown to diminish muscle power across the ankle joint leading to negative changes in the triceps surae musculature [8]. To compensate for this, the hip flexors become the dominant muscle group propelling an individual through ambulation. Dillon, et al. showed that amputation of toes only resulted in no change in the ability to generate power around the ankle joint [8]. Preserving the metatarsal heads is thought to allow concentric contracture of the ankle plantar flexors and normal ankle power [9]. Several studies suggest that only amputations at the level of the level of the MTPJ preserve the use of the ankle joint motion and the calf musculature during gait [10-12]. Mann, et al. reported in 10 patients who underwent hallux amputation there was no difference in cadence, step length, stance phase

Citation: Stowers JM, Carroll P, Sharpe JJ (2020) Preserving Foot Length: Pan-Digital Amputation an Alternative Amputation to Transmetatarsal Amputation: A Case Study. Int J Foot Ankle 4:045. doi. org/10.23937/2643-3885/1710045

Accepted: May 23, 2020: Published: May 25, 2020

Copyright: (c) 2020 Stowers JM, et al. This is an open-access article distributed under the terms of the Creative Commons Attribution License, which permits unrestricted use, distribution, and reproduction in any medium, provided the original author and source are credited. 


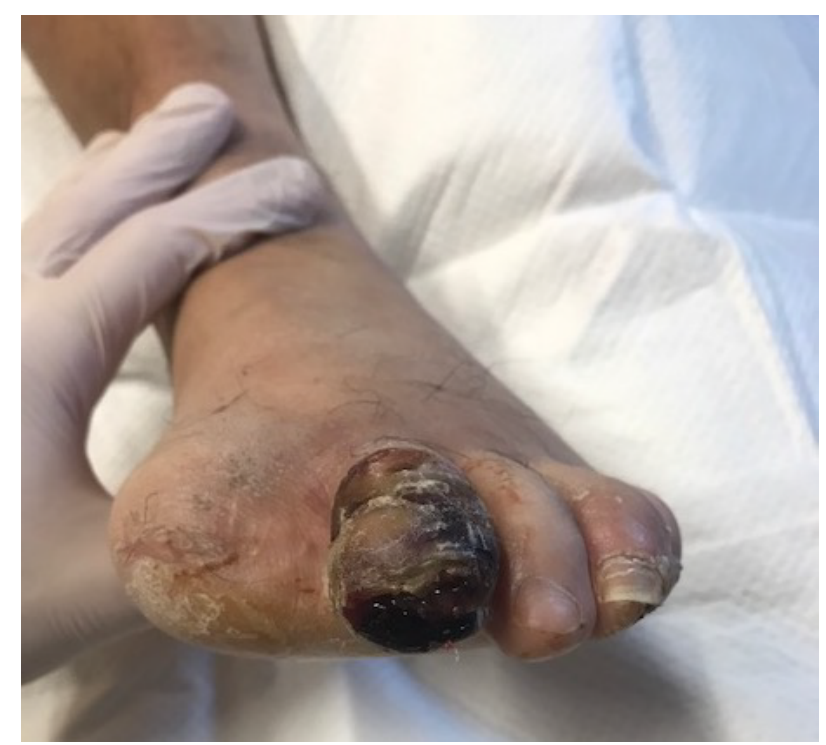

Figure 1: Gangrenous second toe on initial presentation.

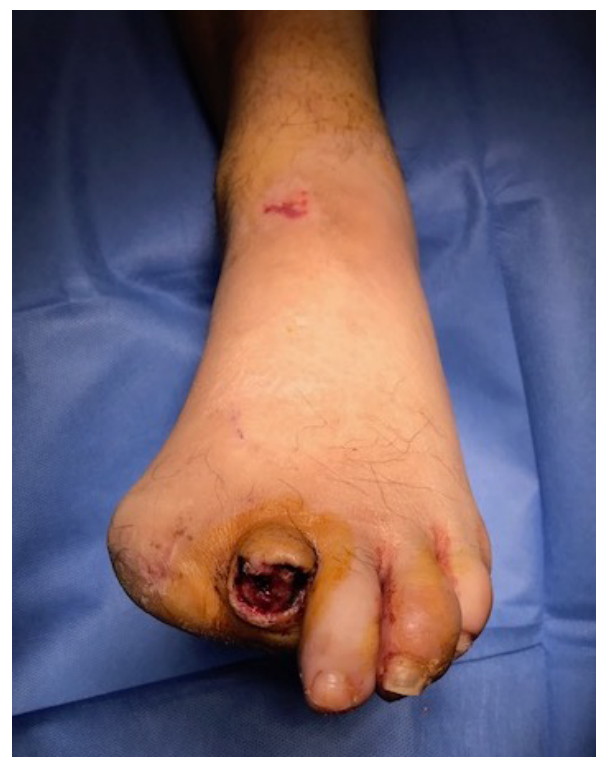

Figure 2: Guillotine amputation of left second toe.

time or swing phase time compared to non-amputees [13].

In certain circumstances a pan-digital amputation may be more advantageous than a transmetatarsal amputation. Without making osteotomies through the metatarsals, preserving length and leaving the articulate cartilage intact this decreases energy consumption as well as decreases pressures points at the distal stumps. Indications for the pan-digital amputation include an unsalvageable forefoot, specifically of the digits, in an ambulatory individual. Disruption of the soft tissue on the plantar metatarsal head area or infection of the metatarsals excludes this type of amputation from consideration.

To our knowledge, there is no published literature discussing a pan-digital amputation. We present the case of a patient who underwent a pan digital ampu-

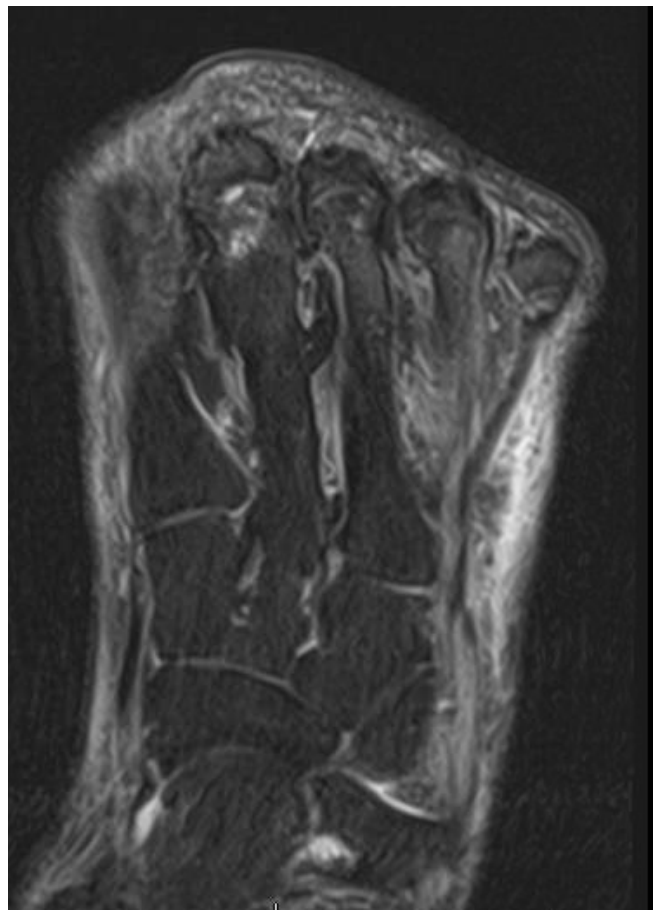

Figure 3: T-2 weighted magnetic resonance imaging (MRI) demonstrating minimal bone marrow edema of the metatarsal heads with no indication of osteomyelitis.

tation after a hallux and lesser digit amputations with recurrent digital pathology.

\section{Case Report}

A 48-year-old male presented to our emergency department in October 2017 with a one-week history of an infected gangrenous left second toe (Figure 1). His past medical history was significant for diabetes, coronary artery disease and peripheral vascular disease. He had undergone a previous hallux amputation on the same foot. Upon admission, his labs and vitals were normal.

Radiograph were negative for gas gangrene, however, was suggestive for osteomyelitis of the second toe. The patient underwent an open guillotine amputation at the interphalangeal joint (Figure 2). An MRI of the forefoot post operatively showed that the metatarsals were unlikely to be infected with osteomyelitis thus no need for further resection (Figure 3). Prior to the final procedure, an in-depth discussion was had with the patient regarding amputation of all remaining toes. It was advised to the patient, leaving the lateral three toes would leave the patient at risk of developing new ulcerations which could become infected. It was recommended to the patient to undergo amputation of the remaining toes and a tendoachilles lengthening (TAL). A standard fish mouth incision pan-digital amputation, with a prophylactic tendoachilles lengthening was performed (Figure 4). The patient's post-operative recovery was uneventful and healed without complication. The patient was ambulating with little difficulty without a prosthetic in sneakers at the most recent clinic visit (Figure 5). 


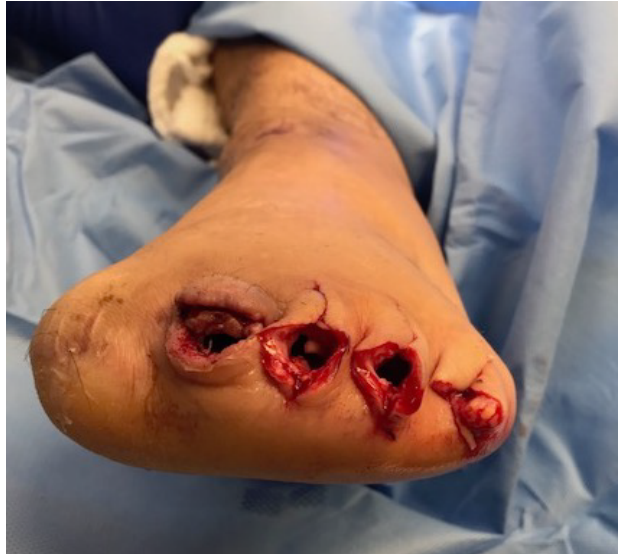

A)

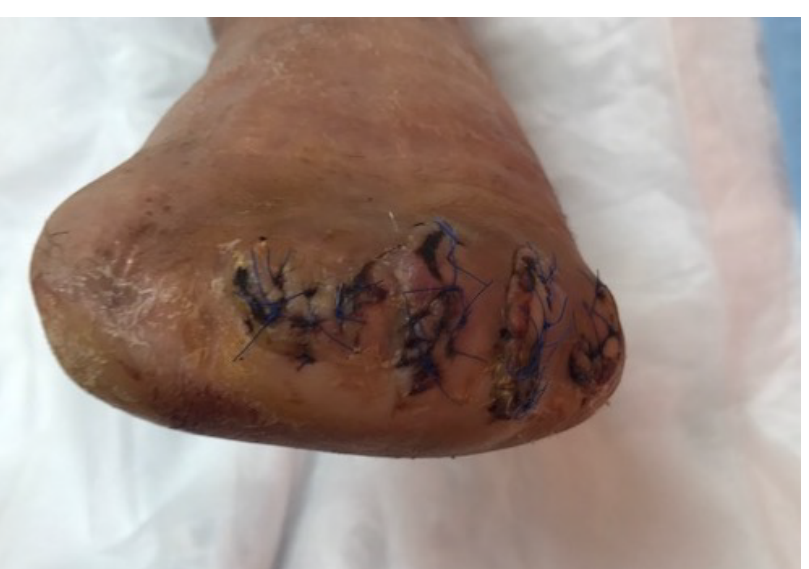

B)

Figure 4: A) Pan digital amputation after disarticulation of all lesser toes; B) Final closure of the amputation.

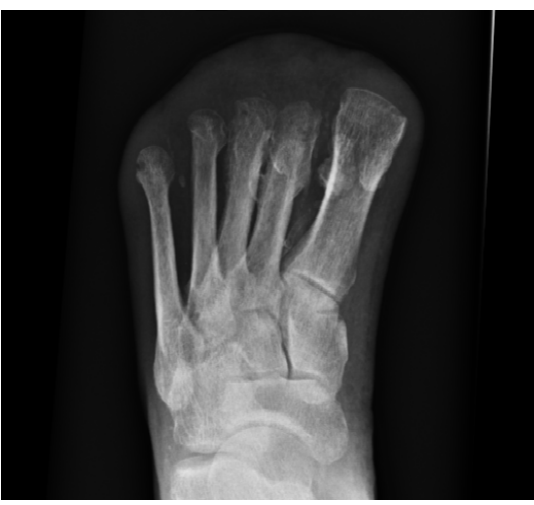

A)

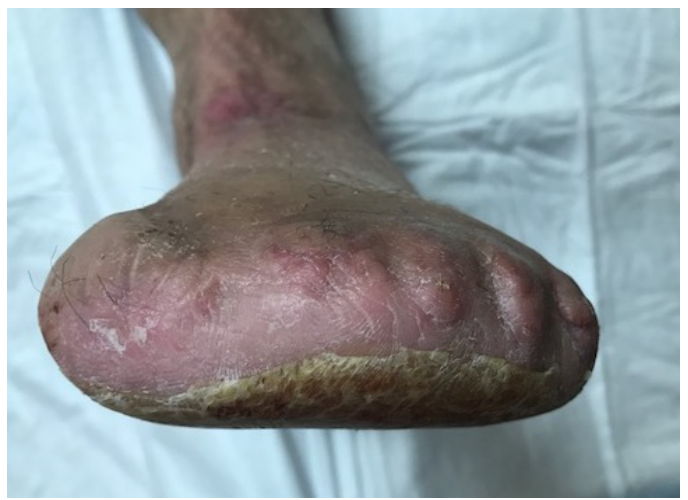

B)

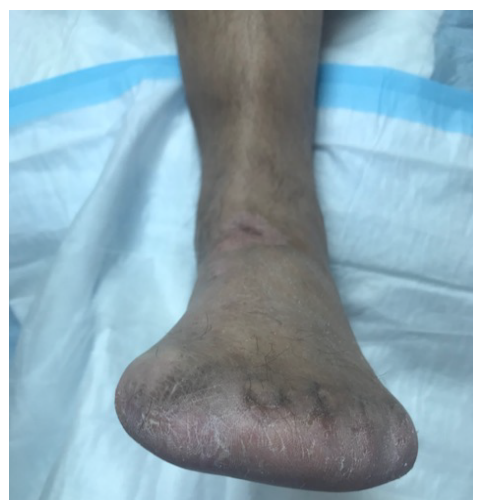

C)

Figure 5 (A-C): Radiographs and clinical photos 9 months after final closure.

\section{Discussion}

The indications for a pan-digital amputation may be limited; however, it may be a more desirable option than a TMA in the appropriate setting. Surgeons may be quick to decide on performing a TMA in the setting of an unsalvageable distal forefoot without fully examining the advantages of preserving foot length. In our patient, it was determined that maintaining foot length in the setting of non-infected metatarsals was the best course of action.

Our patient had a previous hallux amputation which likely led to the development of rigid lesser digit hammertoes. The hammering of his digits led to an infected wound on the distal tip of his second toe. Leaving one or two digits puts the patient at higher risk of trauma and other complications being that studies have shown that $10 \%$ of lesser digits ulcerate in the setting of single digit amputations [4]. This concept was discussed with the patient and helped to guide our decision as to the level of amputation.

As with a TMA, the development of an equinovarus deformities common with a pan-digital amputation and this may lead to higher stress on the distal lateral stump. With the loss of the extensor tendons, the gastroc-soleus complex and tibialis posterior gain mechanical advantage and pull the foot into an equinovarus position $[14,15]$. We proceeded with the prophylactic TAL in anticipation of this biomechanical deformity. The patient had no pressure lesions noted post-operatively or on future clinic visits.

\section{Conclusion}

In the appropriate setting, a pan-digital amputation is an alternative to a TMA. Preserving length decreases energy expenditure and leaving articulate cartilage intact decreases pressure points the amputation. The pan-digital amputation is a viable alternative to the TMA when previous digital amputation, digital deformities, chronic wounds, and osteomyelitis need surgical intervention. With these advantages, this procedure has the potential to maintain ambulation and decrease repeat visits to the operating room.

\section{References}

1. Acar E, Kacira BK (2017) Predictors of lower extremity amputation and reamputation associated with the diabetic foot. J Foot Ankle Surg 56: 1218-1222.

2. McCallum R, Tagoe M (2012) Transmetatarsal amputa- 
tion: A case series and review of the literature. J Aging Res 2012: 797218.

3. Satterfield K (2003) Amputation considerations and energy expenditures in the diabetic patient. Clin Podiatr Med Surg 20: 793-801.

4. Rosen RC (2005) Digital amputations. Clin Podiatr Med Surg 22: 343-363.

5. Mueller MJ, Allen BT, Sinacore DR (1995) Incidence of skin breakdown and higher amputation after transmetatarsal amputation: Implications for rehabilitation. Arch Phys Med Rehabil 76: 50-54.

6. Huang CT, Jackson JR, Moore NB, Fine PR, Kuhlemeier $\mathrm{KV}$, et al. (1979) Amputation: Energy cost of ambulation. Arch Phys Med Rehabil 60: 18-24.

7. Dillon MP, Fatone S (2013) Deliberations about the functional benefits and complications of partial foot amputations: Do we pay heed to the purported benefits at the expense of minimizing complications? Arch Phys Med Rehabil 94: 1429-1435.

8. Dillon MP, Barker TM (2006) Preservation of residual foot length in partial foot amputation: A biomechanical analysis. Foot Ankle Int 27: 110-116.

9. Dillon MP, Barker TM (2008) Comparison of gait of persons with partial foot amputations wearing prosthesis to matched control group: Observational study. J Rehabil Res Dev 45: 1317-1334.

10. Dillon MP (2001) Biomechanical models for the analysis of partial foot amputee gait. Queensland University of Technology, Brisbane.

11. Burger H, Ezar D, Maver T, Olensek A, Cikajlo I, et al. (2009) Biomechanics walking with silicone prosthesis after midtarsal (Chopart) disarticulation. Clin Biomech 24: 510516.

12. Mueller MJ, Salsich GB, Bastian AJ (1998) Differences in the gait characteristics of people with diabetes and transmetatarsal amputation compared with age-matched controls. Gait Posture 7: 200-206.

13. Mann RA, Poppen NK, O'Konski M (1988) Amputation of the great toe. A clinical and biomechanical study. Clin Orthop Relat Res 226: 192-205.

14. Armstrong DG, Stacpoole-Shea S, Nguyen H, Harkless LB (1999) Lengthening of the Achilles tendon in diabetic patients who are at high risk for ulceration of the foot. J Bone Joint Surg Am 81: 535-538.

15. Attinger C, Venturi M, Kim K, Ribiero C (2003) Maximizing the length and optimizing biomechanics in foot amputations by avoiding cookbook recipes for amputation. Semin Vasc Surg 16: 44-66. 